\title{
Análise da Afluência Anual a um Serviço de Atendimento a Situações de Urgência (SASU) do Grande Porto
}

Alves $\mathrm{P}^{*}$, Teixeira Pinto $\mathrm{S}^{*}$, Chaves e Castro $\mathrm{I}^{* * *}$, Pinto $\mathrm{E}^{* * *}$, Valente $\mathrm{C}^{* * * *}$, Alves $\mathrm{L}^{* * * * *}$

\section{Pontos-chave:}

Existe uma variação sazonal na procura de cuidados de saúde urgentes.

Existem fatores socioculturais que influenciam procura de cuidados de saúde urgentes.

O SASU parece resolver a maioria das situações que aí se apresentam sem necessidade de referenciação a níveis de cuidados superiores.

\section{Resumo}

Introdução: A crescente sobrecarga da procura de cuidados de saúde urgentes pela população levou à criação de Serviços de Atendimento a Situações Urgentes (SASU). O seu objetivo é a melhoria da acessibilidade da população e também evitar a deslocação desnecessária de utentes a cuidados de saúde de segunda linha. O objetivo deste estudo é analisar a afluência anual a um SASU do Grande Porto e perceber quais os fatores associados à sua variação.

Metodologia: Realizou-se um estudo descritivo, analítico retrospetivo relativo a todos os episódios de urgência ocorridos durante o ano de 2011 no SASU de Vila Nova de Gaia, tendo em conta as características sociodemográficas dos utentes, a hora do atendimento e a sua orientação após a alta. Estes dados foram analisados cronologicamente, tendo em atenção a época de surto gripal e eventos socioculturais. Foi determinada a cadência diária que representa o número de consultas por médico-hora.

Resultados: Da análise da amostra é possível verificar que globalmente a maioria dos episódios são de utentes do sexo feminino. Apesar disso, na faixa etária inferior a
15 anos, a mais prevalente, o sexo masculino é maioritário. Menos de $2 \%$ dos episódios terminaram com uma referenciação para cuidados hospitalares. Analisando os dados da cadência de episódios existe uma diferença com significado estatístico entre a segunda-feira e o sábado (4,4 vs. 3,3 consultas/médico-hora), assim como entre a segunda-feira e a sexta-feira (4,4 vs. 3,9); a nível mensal no mês de janeiro verifica-se uma cadência superior àquela do mês de agosto em 2 consultas/médico-hora. No Verão ocorreu em média menos 1 consulta/médico-hora do que no Inverno. 0 período de surto gripal foi acompanhado de mais 0,5 consultas/ médico-hora relativamente ao período de menor atividade gripal $(\mathrm{t}(358)=-6,45 ; \mathrm{p}<0,0001)$. Nos dias em que houve jogo do Futebol Clube do Porto verificou-se uma cadência de consultas inferior à verificada em dias sem jogo $(t(358)=27,33 ; p=0,018)$

Discussão: É possível perceber que um dos objetivos de existência do SASU se encontra cumprido, visto que permite uma "desmobilização" virtual de utentes que se dirigiriam de outra forma aos Serviços de Urgência hospitalares. As análises da variação sazonal, epidemiológica e sociocultural dos episódios de urgência vem

\footnotetext{
* Interno Medicina Geral e Familiar, USF St. André de Canidelo

** Diretora Executiva Agrupamento de Centros de Saúde Grande Porto VII-Gaia

*** Presidente Conselho Clínico Agrupamento de Centros de Saúde Grande Porto VII-Gaia

${ }^{* * * *}$ Vogal Conselho Clínico Agrupamento de Centros de Saúde Grande Porto VII-Gaia

${ }^{* * * * *}$ Assistente Medicina Geral e Familiar, USF St. André de Canidelo
} 
reforçar a percepção dos próprios profissionais de saúde e os dados da literatura. Ainda que se possa atribuir um peso elevado à gripe como doença com epidemiologia sazonal, a variação verificada nos diferentes dias de semana parece ser um fator dificilmente explicável por essa razão. A análise dos dados recolhidos e a metodologia utilizada poderão ser ferramentas úteis para serem adotadas em estudos posteriores versando tanto o comportamento da população na utilização do serviço de urgência assim como a melhor forma de organização destes serviços. Alinhando a oferta de cuidados com a procura dos mesmos, conseguir-se-á uma melhor eficiência dos serviços e satisfação tanto dos prestadores como dos utilizadores.

Palavras-chave: sazonalidade, SASU, urgência, afluência

\section{Introdução}

A prestação de cuidados de saúde em situações urgentes é um dos pilares vitais de qualquer sistema de saúde integrado. Em Portugal existem vários níveis de atendimento urgente de complexidade crescente, desde Serviços de Atendimento Permanente, Serviços de Urgência Básica, Serviços de Urgência Médico-Cirúrgica e Serviços de Urgência Polivalente. Esta organização de serviços em graus de crescente complexidade é uma tentativa da tutela orientar a oferta de serviços para a procura de cuidados adequada. Porém, o que se verifica é que uma grande parte dos motivos que levam os utentes ao serviço de urgência prende-se com situações de doença pouco urgentes ou mesmo não urgentes10, beneficiando estes de um acesso facilitado e imediato ao sistema de saúde. Nesse sentido, e visando evitar a deslocação desnecessária dos utentes e a decorrente sobrecarga sobre os serviços de urgência hospitalares, foram criados os Serviços de Atendimento a Situações de Urgência (SASU) na dependência dos Agrupamentos de Centros de Saúde (ACeS). Estes operam em complementaridade com as Unidades de Prestação de Cuidados de Saúde Personalizados (UCSP) e Unidades de Saúde Familiar (USF) de cada ACeS. O alargamento do horário conseguido por estes serviços pretende "desviar" dos serviços de urgência de nível mais diferenciado as situações menos complexas, funcionando como uma continuação dos serviços de atendimento urgente existentes nas unidades de cuidados de saúde primários locais. Isto é perceptível pelas valências que existem no SASU e pelos profissionais que aí trabalham, frequentemente médicos de medicina geral e familiar. No ano de 2011, verificou-se uma alteração organizacional em termos de horas de funcionamento do SASU de Vila Nova de Gaia (encurtamento para 3 horas diárias durante a semana) e com a diminuição do número de médicos nas equipas a partir de Março. Nos anos recentes, tem-se observado uma tentativa de reorganização da rede de atendimento a situações urgentes e emergentes com o encerramento de unidades consideradas redundantes por critérios geográficos e de excesso de oferta. A diminuição da oferta de cuidados deste tipo não se acompanhou de um aumento franco do número de atendimentos a nível hospitalar, o que reforça a noção de redundância da sua existência ${ }^{10}$. Estima-se que o valor de referência de episódios anuais de atendimento na Rede Nacional de Emergência e Urgência seja de 25/100 habitantes.

A procura por cuidados de saúde de urgência é influenciada por complexos fatores muitas vezes observados e referidos de forma empírica pelos profissionais que aí trabalham. Estão descritas também associações entre o número de episódios de urgência e fatores como idade, dias da semana, meses e estações do ano, temperatura ambiente, precipitação $0^{1,3}$, ocorrência de eventos socioculturais de relevo ${ }^{2,7}$ e épocas festivas. 0 caso dos dias em que existe um desafio desportivo é um dos mais mencionados, apesar de existirem evidências contraditórias na literatura $2,4,5,6$.

O objetivo deste estudo é analisar num período de um ano a afluência a um Serviço de Atendimento a Situações de Urgência e perceber qual a variação existente em relação com aspetos sazonais, epidemiológicos e socioculturais.

\section{Métodos}

Realizou-se um estudo observacional, descritivo e analítico retrospectivo através da recolha de informação fornecida pelo ACeS com conhecimento e consentimento da Direcção Executiva do ACeS, referente a todos os episódios de consulta registados no SASU de Vila Nova de Gaia, ocorridos entre os dias 01/01/2011 e 31/12/2011. Cada episódio de urgência foi identificado através da data, hora de atendimento, sexo, idade do utente e destino após o atendimento. Através da análise da data de cada episódio, foi possível criar novas variáveis referentes ao dia da semana (domingo, segunda-feira, terça-feira, quarta-feira, quinta-feira, sexta-feira e sábado), mês (janeiro, fevereiro, março, abril, 
maio, junho, julho, agosto, setembro, outubro, novembro e dezembro), estação do ano (inverno, primavera, verão e outono).

A época de surto gripal ativo foi determinada através dos relatórios de saúde pública nacionais, correspondendo às primeiras doze e às últimas doze semanas desse $a n 0^{8,9}$. Daqui resultou uma variável dicotómica definida pela presença ou ausência de atividade gripal no período em questão. O destino dos utentes após avaliação médica dividiu-se nas seguintes categorias: Ambulatório; Domicílio/Alta; Em tratamento; Cuidados hospitalares; Internamento; Desistência e Falecido, segundo aquilo que o médico considerou no sistema informático. Estas categorias foram agregadas na variável destino: Ambulatório/Domicílio/Alta; Cuidados hospitalares (agregando as categorias Cuidados Hospitalares e Internamento da variável original); Falecido; Outros (agregando as categorias Desistência e Em tratamento da variável original). Foi criada a variável dicotómica Jogos Futebol Clube do Porto (FCP) tendo em conta a ocorrência no dia analisado de jogos de futebol em que participasse a equipa sénior do Futebol Clube do Porto ${ }^{12}$.

Foi contabilizado o número total de consultas em cada dia de funcionamento do SASU. Para cada dia, contabilizou-se o número de profissionais médicos ao serviço, bem como o número de horas de funcionamento do SASU. Esta análise considerou os dias de fim de semana e os dias em que foi feriado, assim como as variações ao longo do ano no número de horas de atendimento no SASU e constituição das respetivas equipas médicas. Como indicador da capacidade diária de atendimento no SASU, criou-se a variável médico-hora, obtida do produto do número de médicos ao serviço pelas horas de atendimento no SASU, em cada dia de funcionamento. A variável cadência decorreu da conjugação do número de consultas diárias com a variável médico-hora de forma a conseguirmos obter um indicador da produtividade independente do número de médicos ou horário de funcionamento.

As variáveis categóricas e contínuas foram apresentadas por contagem (percentagem) e pela média (desvio-padrão), respetivamente. A normalidade da distribuição das amostras foi avaliada através de testes Shapiro-Wilk. A comparação de médias de cadência foi realizada com recurso a testes $t$-student ( $\alpha=95 \%$ ) e ao critério de não sobreposição de intervalos de confiança para validação da significância estatística. Os dados foram recolhidos em formato Microsoft ${ }^{\circledR}$ Excel $^{\circledR}$, sendo posteriormente tratados em Stata ${ }^{\circledR}$.

\section{Resultados}

O número total de atendimentos realizados no período em análise foi de 36060 episódios.

Na tabela 1 é perceptível uma maior afluência da parte de utentes do sexo feminino relativamente ao sexo masculino no global. Esta tendência torna-se notória nas faixas etárias acima dos 15 anos, verificando-se uma inversão do rácio em jovens com menos de 15 anos de idade. Existe um grande peso da população pediátrica nos episódios de SASU, com a maior prevalência de episódios a acontecer em jovens com menos de 15 anos, independentemente do sexo. 0 maior volume de episódios de urgência encontra-se concentrado nos horários das 21 às 23 horas dado ser este o horário em que o SASU está aberto, independentemente do dia da semana.

Analisando os dados relativos à orientação dada aos utentes que procuram o SASU, é possível perceber que apenas numa minoria dos casos existe referenciação direta a cuidados hospitalares, tanto em homens como em mulheres.

Numa perspetiva global e utilizando a variável cadência referente ao número de consultas por médico-hora por dia ao longo do ano, podemos observar que existe uma tendência representada por curva de concavidade superior com valores mais elevados tanto no início como no final do ano [Gráfico 1]. A cadência diária média $( \pm \sigma)$ é de $3,82( \pm 0,79)$ consultas/médico-hora com valores máximos de 6,10 consultas/médico-hora e mínimos de 1,63 consultas/médico-hora. De notar que em alguns dias os valores de cadência foram francamente díspares da tendência global, verificando-se menos de 2 consultas por médico-hora nos dias 23 de Junho e dia 24 de Dezembro. Quando avaliamos o número de consultas/médico-hora em termos da sua ocorrência ao longo da semana é perceptível uma diferença estatisticamente significativa entre a segunda-feira e a sexta-feira [cadência média (IC95\%): 4,4 (4,2-4,6) vs. $3,9(3,7-4,1)]$, representada por menos 0,5 consultas/ médico-hora. Quando comparamos o número médio de consultas/médico-hora à segunda-feira e ao sábado, verifica-se uma diferença com significância estatística superior a 1 consulta/médico-hora [cadência média (IC95\%): 4,4 (4,2-4,6) vs. 3,3 (3,2-3,4)] [Gráfico 2]. Da análise mensal, ressalta a diferença entre os meses de janeiro e agosto [cadência média (IC95\%): 4,7 (4,4$5,1)$ vs. 3,0 (2,9-3,2)], verificando-se uma diminuição de quase menos 2 consultas/médico-hora [Gráfico 3]. Em termos sazonais, há entre o Inverno e o Verão 
uma diferença estatisticamente significativa de menos 1 consulta/médico-hora em média [cadência média (IC95\%): 4,2 (4,0-4,3) vs. 3,3 (3,2-3,4)] [Gráfico 4]. Relativamente à época de atividade gripal, é possível verificar que existe uma diferença em termos de número médio de consultas/médico-hora, com mais 0,5 consultas/médico-hora durante os períodos de atividade gripal $(\mathrm{t}(358)=-6,45 ; \mathrm{p}<.0001)$.

Analisando a cadência média em dias em que há jogo do FCP e dias em que não há, obtemos uma diferença com significância estatística com um valor médio mais elevado nos dias em que não há jogo (t(358)=2,37; $p=0,018)$ [Gráfico 6].

Tabela 1: Descrição da amostra

\begin{tabular}{|c|c|c|}
\hline & Mulheres $\mathrm{N}^{\circ}(\%)$ & Homens $\mathrm{N}^{\circ}(\%)$ \\
\hline Episódios & $21988(100,0)$ & $14072(100,0)$ \\
\hline \multicolumn{3}{|l|}{ Por faixa etária } \\
\hline Menos 15 anos & $5268(24,0)$ & $5339(37,9)$ \\
\hline $15-29$ anos & $4498(20,5)$ & $2354(16,7)$ \\
\hline 30-44 anos & $5132(23,3)$ & $2668(19,0)$ \\
\hline 45-59 anos & $4046(18,4)$ & $2073(14,7)$ \\
\hline 60-74 anos & $2064(9,4)$ & $1192(8,5)$ \\
\hline 75 anos ou mais & $980(4,5)$ & $446(3,2)$ \\
\hline \multicolumn{3}{|l|}{ Por destino } \\
\hline Ambulatório & $4996(22,72)$ & $3236(23,0)$ \\
\hline Domicílio/Alta & $16679(75,86)$ & $10572(75,13)$ \\
\hline Cuidados hospitalares & $300(1,36)$ & $259(1,84)$ \\
\hline Falecido & $1(0,0)$ & $0(0,0)$ \\
\hline Outros & $12(0,05)$ & $5(0,04)$ \\
\hline \multicolumn{3}{|l|}{ Por horário de inscrição } \\
\hline $9 h-12 h$ & $3232(14,7)$ & $2166(15,39)$ \\
\hline $12-15 \mathrm{~h}$ & $2616(11,9)$ & $1586(11,27)$ \\
\hline $15 h-18 h$ & $3225(14,67)$ & $2038(14,48)$ \\
\hline $18 \mathrm{~h}-21 \mathrm{~h}$ & $5855(26,63)$ & $3610(25,65)$ \\
\hline $21 \mathrm{~h}-23 \mathrm{~h}$ & $7060(32,11)$ & $4672(33,2)$ \\
\hline \multicolumn{3}{|l|}{ Por trimestre } \\
\hline Jan-Mar & $6464(29,4)$ & $4298(30,54)$ \\
\hline Abr-Jun & $5416(24,63)$ & $3491(24,81)$ \\
\hline Jul-Set & $4574(20,8)$ & $2820(20,04)$ \\
\hline Out-Dez & $5534(25,17)$ & $3463(24,61)$ \\
\hline
\end{tabular}


Tabela 2: Atividade do SASU Vila Nova de Gaia durante o ano de 2011

\begin{tabular}{|c|c|c|c|c|c|c|}
\hline & Consultas $\mathbf{N}^{\circ}$ & $\begin{array}{l}\text { Consultas/dia } \\
\text { Média (dp) }\end{array}$ & $\begin{array}{l}\text { Horas/dia de } \\
\text { funcionamento } \\
\text { Média (dp) }\end{array}$ & $\begin{array}{l}\text { Consultas por } \\
\text { hora } \\
\text { Média }(\mathrm{dp})\end{array}$ & $\begin{array}{l}\text { Cadência - Consultas } \\
\text { por médico-hora } \\
\text { Média }(\mathrm{dp})\end{array}$ & IC95\% \\
\hline \multicolumn{7}{|l|}{ Dia da semana } \\
\hline Domingo & 8869 & $177,4(36,0)$ & $13,4(0,9)$ & $13,3(2,5)$ & $3,2(0,5)$ & $3,0-3,3$ \\
\hline Segunda-feira & 3731 & $71,8(23,2)$ & $3,4(2,1)$ & $22,2(4,0)$ & $4,4(0,8)$ & $4,2-4,6$ \\
\hline Terça-feira & 3375 & $67,5(25,9)$ & $3,4(2,0)$ & $20,7(4,1)$ & $4,1(0,8)$ & $3,8-4,3$ \\
\hline Quarta-feira & 3216 & $63,1(20,8)$ & $3,2(1,3)$ & $20,1(3,8)$ & $4,0(0,7)$ & $3,8-4,2$ \\
\hline Quinta-feira & 3524 & $67,8(27,1)$ & $3,6(2,3)$ & $20,3(4,5)$ & $4,0(0,9)$ & $3,7-4,2$ \\
\hline Sexta-feira & 3493 & $67,2(29,9)$ & $3,6(2,6)$ & $19,7(3,9)$ & $3,9(0,8)$ & $3,7-4,1$ \\
\hline Sábado & 9852 & $185,9(31,9)$ & $13,4(0,9)$ & $13,9(2,1)$ & $3,3(0,4)$ & $3,2-3,4$ \\
\hline \multicolumn{7}{|l|}{ Mês } \\
\hline Janeiro & 4096 & $132,1(77,6)$ & $6,5(5,2)$ & $23,7(5,0)$ & $4,7(1,0)$ & $4,4-5,1$ \\
\hline Fevereiro & 3246 & $115,9(70,4)$ & $6,1(5,1)$ & $21,9(4,8)$ & $3,8(0,6)$ & $3,6-4,0$ \\
\hline Março & 3420 & $110,3(65,7)$ & $6,2(5,1)$ & $20,8(4,3)$ & $4,4(0,6)$ & $4,1-4,6$ \\
\hline Abril & 3293 & $113,6(63,7)$ & $7,2(5,4)$ & $18,9(4,8)$ & $4,0(0,8)$ & $3,7-4,3$ \\
\hline Maio & 2806 & $93,5(50,7)$ & $6,3(5,1)$ & $18,0(5,0)$ & $3,8(0,8)$ & $3,5-4,1$ \\
\hline Junho & 2808 & $96,8(96,8)$ & $6,8(5,3)$ & $17,2(4,4)$ & $3,6(0,7)$ & $3,3-3,9$ \\
\hline Julho & 2602 & $86,7(50,5)$ & $6,3(5,1)$ & $16,2(3,8)$ & $3,4(0,6)$ & $3,2-3,6$ \\
\hline Agosto & 2374 & $79,1(49,8)$ & $6,3(5,1)$ & $14,4(3,0)$ & $3,0(0,4)$ & $2,9-3,2$ \\
\hline Setembro & 2418 & $80,6(45,6)$ & $5,5(4,3)$ & $16,5(3,2)$ & $3,5(0,5)$ & $3,3-3,6$ \\
\hline Outubro & 3150 & $101,6(55,4)$ & $6,2(4,4)$ & $18,5(3,8)$ & $3,9(0,6)$ & $3,7-4,1$ \\
\hline Novembro & 2852 & $95,1(51,4)$ & $5,7(4,2)$ & $18,9(4,1)$ & $4,0(0,6)$ & $3,7-4,2$ \\
\hline Dezembro & 2995 & $96,6(50,9)$ & $6,2(4,4)$ & $18,0(4,2)$ & $3,8(0,7)$ & $3,5-4,0$ \\
\hline \multicolumn{7}{|l|}{ Estação } \\
\hline Inverno & 12066 & $112,8(67,3)$ & $6,2(4,9)$ & $21(5,1)$ & $4,2(0,9)$ & $4,0-4,3$ \\
\hline Primavera & 9347 & $102,7(57,2)$ & $6,6(5,2)$ & $19(4,8)$ & $3,9(0,8)$ & $3,8-4,1$ \\
\hline Verão & 7562 & $83,1(48,9)$ & $6,2(4,9)$ & $16(3,7)$ & $3,3(0,6)$ & $3,2-3,4$ \\
\hline Inverno & 6853 & $97,9(53,0)$ & $6,0(4,3)$ & $19(3,8)$ & $3,9(0,6)$ & $3,8-4,0$ \\
\hline
\end{tabular}

Gráfico 1: Cadência diária no SASU em 2011

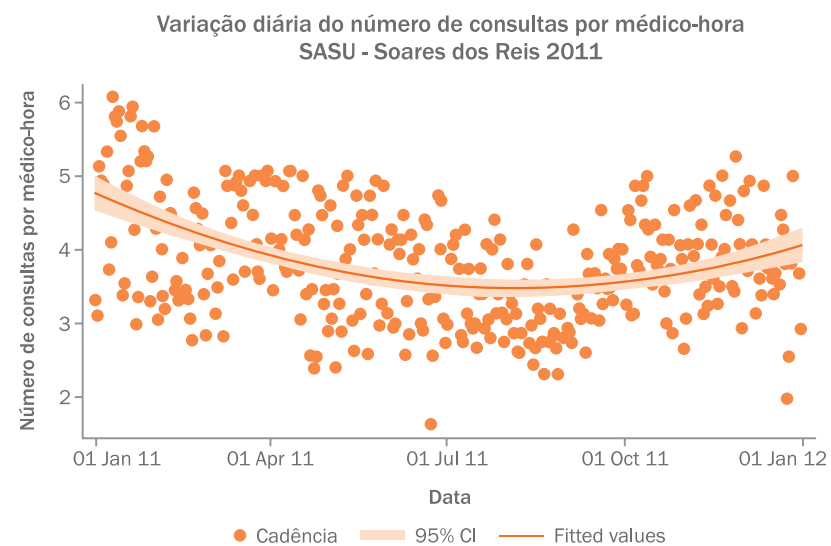

Gráfico 2: Cadência por dia da semana

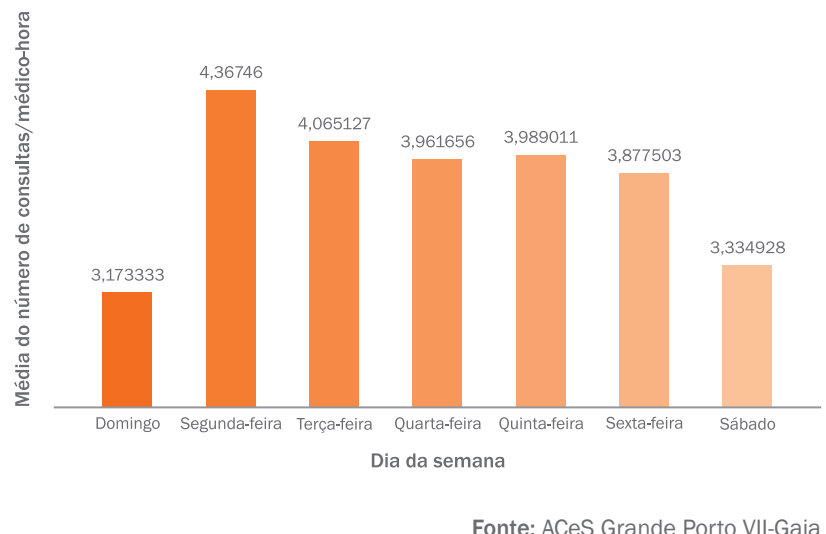


Gráfico 3: Cadência mensal

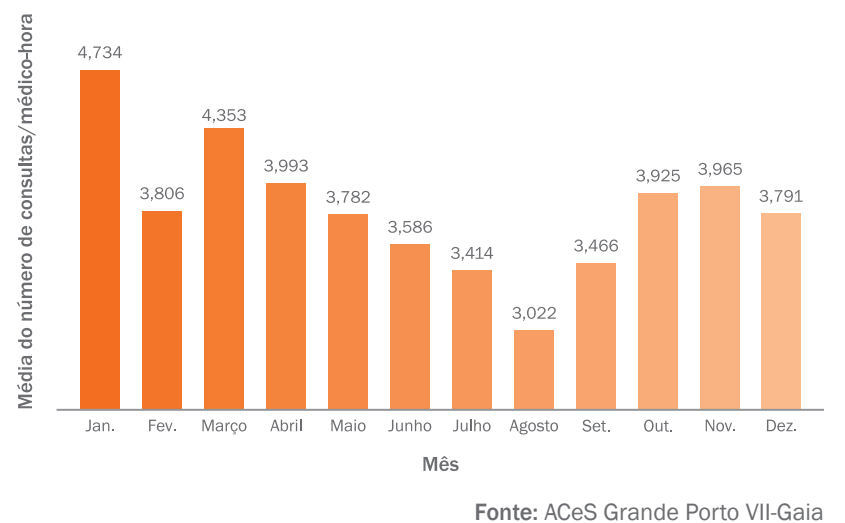

Gráfico 4: Cadência por estação do ano

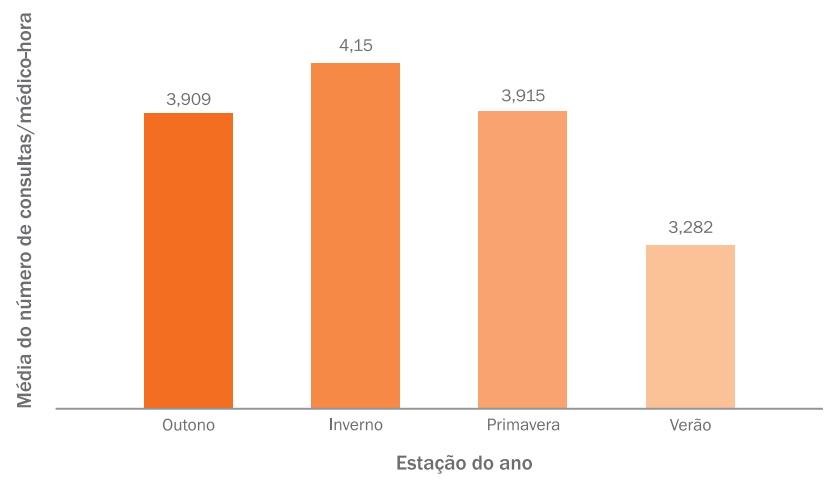

Fonte: ACeS Grande Porto VII-Gaia

Gráfico 5: Cadência média em época de surto gripal

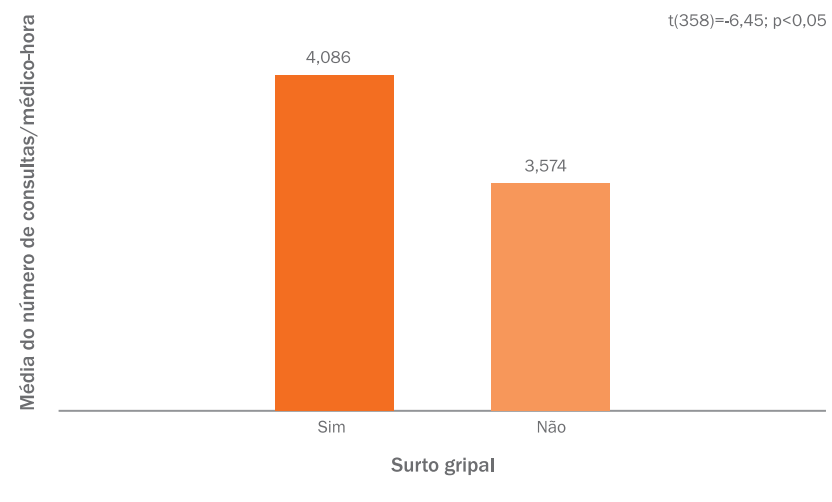

Fonte: ACeS Grande Porto VII-Gaia
Gráfico 6: Cadência média consoante a ocorrência de jogos do Futebol Clube do Porto

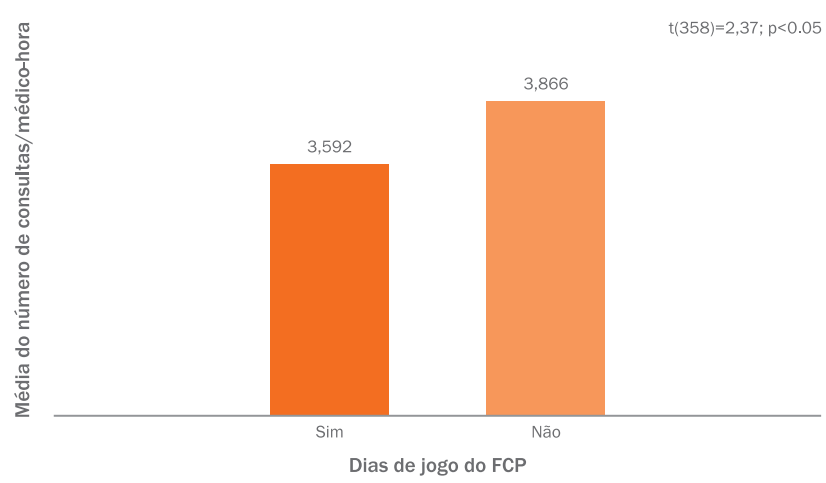

Fonte: ACeS Grande Porto VII-Gaia

\section{Discussão}

Da análise realizada e através dos resultados obtidos, é possivel verificar uma associação entre a afluência dos utentes ao SASU e aspetos de ordem sazonal, epidemiológico e sociocultural com especial ênfase para o efeito "segunda-feira" como pico semanal de atendimentos e a reduzida afluência verificada nos meses de verão.

A população que acorre ao SASU é tendencialmente jovem, com idade inferior a 15 anos e maioritariamente do sexo feminino. São sujeitos a referenciação direta para cuidados hospitalares uma minoria da população assistida o que parece ir de encontro a um dos objetivo da existência deste serviço: servir como triagem e solução para problemas que não necessitam de cuidados de segunda ou terceira linha. A noção empírica que os profissionais têm da carga de procura dos cuidados de saúde é validada pelos dados que revelam que nos meses de inverno e outono e durante o surto gripal existe um acréscimo da cadência de consultas. Numa perspetiva semanal, independentemente do mês ou altura em questão é curioso verificar a afluência tendo em conta o fim de semana e o início da semana e a forma como as pessoas vão procurando cada vez menos os serviços de segunda-feira a sexta-feira. Os valores de cadência nas datas referentes à véspera do feriado municipal de São João (23 de Junho) e a véspera de do dia de Natal 
(24 de Dezembro) refletem uma tendência já estabelecida de reduzida afluência da população em épocas festivas. Neste estudo constatamos que a ocorrência de um evento desportivo, como o jogo do clube com mais simpatizantes na região, tem impacto dissuasor de recurso ao SASU, facto a que não será alheia a positiva prestação desportiva do clube no ano em questão.

A taxa de atendimentos urgentes na população estudada apresenta um valor aproximado daquele assumido pela tutela como o indicador de garantia de acessibilidade dos utentes a este tipo de cuidados ${ }^{10}$.

As forças desta investigação prendem-se com o facto do estudo abranger um período de análise suficientemente longo, o que permite observar as eventuais variações desejadas. Foi reunida uma amostra grande de observações que contribuiu para a validação estatística das conclusões. São limitados os estudos nacionais e até internacionais nesta área, versando a sua análise mais sobre fatores de risco para as patologias abordadas em contexto de urgência. Os resultados encontrados permitem validar uma metodologia que pode ser adaptada a qualquer tipo de serviço em que a resposta dependa de horas de funcionamento e profissionais ao serviço. A variável cadência permite reunir esses dados e parametrizar serviços com diferentes características num denominador comum e a partir daí poder compará-los. As limitações encontradas neste estudo decorrem principalmente do "efeito de teto". Este efeito advém de um aspeto funcional do SASU que é o habitual encerramento do atendimento assim que se atinja um limite de inscrições de utentes; este limiar é estabelecido pela equipa de profissionais a operar no próprio dia, não sendo estabelecido o critério que leva a que tal aconteça. Estas ocorrências estabelecem um teto de "procura" que pode subestimar as diferenças verificadas. A taxa de referenciação direta a cuidados mais diferenciados encontrada pode estar subestimada, uma vez que não existem dados que nos permitam perceber quantos utentes terão procurado esses cuidados após terem tido alta não referenciada do SASU. A questão da pertinência do recurso aos serviços de atendimento urgente não foi aqui abordada por ausência dos motivos de consulta nos dados disponibilizados, porém seria importante para validar as conclusões do estudo quanto ao comportamento de "procura" pelos utentes.

Ainda que a mera interpretação dos resultados constitua um interessante exercício de epidemiologia e estudo comportamental da população utilizadora dos recursos de urgência, a metodologia deste estudo pode servir como ferramenta importante para a planificação dos serviços e a gestão da oferta de cuidados de saúde à população. A análise retrospetiva dos dados pode e deve ter influência direta na forma como são geridos e disponibilizados os recursos à população em termos de horário de funcionamento, profissionais alocados e recurso a serviços complementares (alargamento dos horários nas Unidades de Saúde Familiar e Unidades de Cuidados de Saúde Primários em períodos críticos).

Por outro lado, a forma como varia o comportamento dos utentes relativamente à necessidade de um atendimento urgente tendo em conta aspetos que não terão relação direta com o estado de saúde merece uma reflexão sobre as motivações dos mesmos e o tipo de resposta que pretendem do sistema através destes contactos (ignorância do conceito de "urgência médica", resposta reduzida dos meios alternativos - linha de atendimento Saúde 24, acessibilidade reduzida ou nula a médico assistente). Novas linhas de estudo deverão abordar estas questões de forma a obtermos um alinhamento mais eficiente entre a procura e a oferta de cuidados de saúde.

\section{Agradecimentos}

Gostaria de agradecer ao Exm. ${ }^{\circ}$ Coordenador da Unidade de Saúde Familiar St. André de Canidelo Dr. Fernando Ferreira. 


\section{Referências Bibliográficas}

1. Atendimento das situações de doença aguda em cuidados de saúde primários e interligação com a Rede Nacional de Emergência e Urgência. Grupo Técnico para o desenvolvimento dos Cuidados de Saúde Primários. Documento de trabalho - versão 30.09.2012. Ministério da Saúde. Disponivel em http://www.acss.min-saude.pt/Portals/0/27interfacesiii-abordagemsistmicadoatendimentodassituaesagudaseurgentes_gt-dcsp_2012.09.30.pdf Acedido a 25.03.2014.

2. Downing A, Wilson R. Temporal and demographic variations in attendance at accident and emergency departments. Emergency Medicine Journal : EMJ 2002; 19:531-535.

3. Furyk JS, Lawlor JM, Franklin R, et al. Emergency department presentations during the State of Origin rugby league series: a retrospective statewide analysis. The Medical Journal of Australia 2012; 197: 663-666.

4. Friede KA, Osborne MC, Erickson DJ, et al. Predicting trauma admissions: the effect of weather, weekday, and other variables. Minn Med. 2009;92:47-49.

5. McGreevy A, Millar L, Murphy B, Davison GW, Brown R, O'Donnell ME. The effect of sporting events on emergency department attendance rates in a district general hospital in Northern Ireland. Int J Clin Pract. 2010;64:1563-1569.

6. Moody WE, Hendry RG, Muscatello D. Were attendances to accident and emergency departments in England and Australia influenced by the Rugby World Cup Final 2003? Eur J Emerg Med. 2007;14:68-71.

\section{Conflito de Interesses}

Os autores declaram não ter conflito de interesses em relação a este artigo.

\section{Endereço para Correspondência:}

Pedro Alves Leite

Email: pedro.alves.leite@gmail.com

USF St. André de Canidelo

Rua das Fábricas, 282

4400-232 Canidelo
7. Cooke MW, Allan TF, Wilson S. A major sporting event does not necessarily mean an increased workload for accident and emergency departments. Euro96 Group of Accident and Emergency Departments. Br J Sports Med. 1999;33:333-335. doi:10.1136/bjsm.33.5.333.

8. Redelmeier DA, Vermeulen MJ. Emergency department visits during an Olympic gold medal television broadcast. Open Med. 2011;5:112-119.

9. Programa Nacional de Vigilância da Gripe - Relatório da época 2010/11. Instituto Nacional de Saúde Doutor Ricardo Jorge, IP. Disponível em: http://repositorio.insa.pt/ bitstream/10400.18/1009/1/Relatorio\%20PNVG\%20 2010-2011.pdf. Acedido a 25.03.2014.

10. Médicos Sentinela: Relatório de Atividades 2011. Instituto Nacional de Saúde Doutor Ricardo Jorge, IP. Disponível em http://repositorio.insa.pt/bitstream/10400.18/1150/3/ relatorio\%20medicos-sentinela\%202011\%20\%20final_ web.pdf. Acedido a 25.03.2014.

11. Plano Local de Saúde 2011-2016 - Agrupamento de Centros de Saúde Grande Porto Gaia VIII. Unidade de Saúde Pública. Disponivel em http://www.cm-gaia.pt/documentos/cmg/ Ac\%C3\%A7aosocial/ACESGAIA.pdf. Acedido a 25.03.2014.

12. Calendário de jogos de futebol da equipa principal do Futebol Clube do Porto. Disponível em http://www.zerozero.pt/equipa.php?id=9\&epoca_id=140\&menu=results; http://www.zerozero.pt/equipa.php?id=9\&epoca_ $i d=141 \&$ menu=results. Acedido a 25.03.2014. 